\title{
Abundance patterns in the galactic bulge: cluster vs field populations
}

\author{
Livia Origlia ${ }^{1}$, R. Michael Rich ${ }^{2}$ and Elena Valenti ${ }^{3}$ \\ ${ }^{1}$ INAF-Osservatorio Astronomico di Bologna, Via Ranzani 1, I-40127 Bologna, Italy \\ email: livia.origlia@bo.astro.it \\ ${ }^{2}$ Department of Physics and Astronomy, University of California at Los Angeles, Los Angeles, \\ CA 90095-1562 \\ email: rmr@astro.ucla.edu \\ ${ }^{3}$ Dipartimento di Astronomia, Università di Bologna, Via Ranzani 1, 40127 Bologna, Italy, \\ email: elena.valenti3@unibo.it
}

\begin{abstract}
Despite its importance, the Galactic Bulge and its globular cluster (GC) system in particular, remain relatively unexplored due to high foreground extinction and severely crowded field populations. The new generation of high resolution IR spectrographs opens new and unique perspectives to obtain detailed abundances in the Bulge field and GCs. Using the NIRSPEC spectrograph at Keck II, high resolution, echelle spectra in the range 1.5-1.8 micron of bright giants in the Baade's window and other fields closer to the Galactic center as well as in a sample of 10 Bulge GCs have been obtained. We present the results of our abundance analysis of Fe, $\mathrm{Al},{ }^{12} \mathrm{C}, 13 \mathrm{C}$, O, and other $\alpha$-elements, by using full spectral synthesis techniques.
\end{abstract}

Keywords. Galaxy: bulge, Galaxy: globular clusters, stars: abundances, infrared:stars

\section{Introduction}

The systematic exploration of the Galactic Bulge started in the 1980's with pioneering work by e.g. Blanco, McCarthy \& Blanco (1984), Frogel \& Whitford (1987), Terndrup, Frogel, \& Whitford (1990), Terndrup, Frogel \& Whitford (1991), Rich (1988) using near infrared (IR) photometry and low resolution spectroscopy. In order to study both the field and GC Bulge population with the necessary statistical significance, near IR observations are crucial, since much less affected by reddening and blanketing effects than optical ones. Moreover, the IR spectral range provides the highest sensitivity to the physical parameters of cool stars and the contrast between the red giants and the fainter Main Sequence population is greater than in any other optical passband, drastically mitigating the problem of image crowding, even in the innermost core region of GCs. Abundance determinations in the Bulge are confounded by the extinction and especially by the high metallicity, which makes abundance analysis very difficult. The most accurate abundance determinations obtained so far and based on high resolution optical spectroscopy refer to a sample of K-giants in the Baade's window (McWilliam \& Rich (1994), Rich \& McWilliam (2000), Fulbright, Rich \& McWilliam (2004)) and a few giants in two GCs, namely NGC 6553 and NGC 6528 (Carretta et al. (2001), Zoccali et al. (2004)). Recently, NGC 6553 (Melèndez et al. (2003)) and Pal 6 (Lee, Carney \& Balachandran (2004)) have been observed at high resolution in the IR using Phoenix at Gemini and CSHELL at the IRTF, respectively. All these studies find some level of $\alpha$-enhancement with respect to solar values both in the Bulge field and GCs. Over the past few years we have commenced a spectroscopic survey of the Galactic Bulge and its GC system, using NIRSPEC, a high throughput infrared (IR) echelle spectrograph at the Keck Observatory (McLean et al. 
(1998)). We derived chemical abundances for six Bulge GCs: the results for NGC 6553 and Liller 1 are given in Origlia, Rich \& Castro (2002), abundances for Terzan 4 and Terzan 5 are reported in Origlia \& Rich (2004), while those for NGC 6342 and NGC 6528 are given in Origlia, Valenti \& Rich (2005). We find $\alpha$-enhancement at a level of a factor between 2 and 3 over the whole range of metallicity spanned by the GCs in our survey, from $[\mathrm{Fe} / \mathrm{H}] \approx-1.6$ (cf. Terzan 4 ) up to $[\mathrm{Fe} / \mathrm{H}] \approx-0.1$ (cf. Terzan 5, NGC 6528). Other 2 GCs, namely NGC 6539 and UKS1 have been recently analyzed (Origlia et al. (2005)), finding $[\mathrm{Fe} / \mathrm{H}] \approx-0.8$ and $\alpha$-enhancement, as in the others. We also reported the first abundance analysis of 14 M giant stars in the Baade's window (Rich \& Origlia (2005)), finding $\mathrm{Fe}$ abundances similar to those of the $\mathrm{K}$ giants as determined from optical high resolution spectroscopy: $[\mathrm{Fe} / \mathrm{H}]=-0.190 \pm 0.020$, with a $1 \sigma$ dispersion of $0.080 \pm 0.015$. Comparing our Bulge $\mathrm{M}$ giants to a control sample of local disk $\mathrm{M}$ giants in the solar vicinity, we find the Bulge stars have $[\alpha / \mathrm{Fe}] \approx+0.3$ dex relative to the solar composition stars, consistent with the studies of Bulge GCs.

In the following we summarize the results of the $\mathrm{Fe}, \mathrm{C}, \mathrm{O}$ and the other $\alpha$-element abundances published so far complemented with preliminary abundances for other $\approx 10$ giants in 2 GCs (NGC 6440 and NGC 6441) and in a field much closer to the Galactic center at $\mathrm{l}=0^{\circ}, \mathrm{b}=-1.0^{\circ}$.

\section{Observations and spectral analysis}

We use NIRSPEC to acquire spectra of cool giants in the $\mathrm{H}$-band $(1.5-1.8 \mu \mathrm{m})$, where most of the atomic and molecular lines of interest are concentrated. The high resolution echelle mode $(\mathrm{R}=25,000)$, with a slit width of $0.43^{\prime \prime}$ (3 pixels) and a length of $12^{\prime \prime}$ and the standard NIRSPEC-5 setting have been selected. Typical exposure times (on source) ranged from 4 to 20 minutes. The raw spectra have been reduced using the REDSPEC IDL-based package written at the UCLA IR Laboratory. Each order has been sky subtracted by using nodding pairs and flat-field corrected. Wavelength calibration has been performed using arc lamps and a 2-nd order polynomial solution, while telluric features have been removed by using an O-star featureless spectrum. At the NIRSPEC resolution of $\mathrm{R}=25,000$ several single roto-vibrational $\mathrm{OH}$ lines and $\mathrm{CO}$ bandheads can be measured to derive accurate $\mathrm{O}$ and $\mathrm{C}$ abundances. Additional metal abundances of $\mathrm{Fe}, \mathrm{Mg}, \mathrm{Si}$, $\mathrm{Ti}$, $\mathrm{Ca}$ and $\mathrm{Al}$ can be derived from neutral atomic lines. Abundance analysis is performed by using full spectral synthesis techniques and equivalent width measurements of representative lines. The latter are computed by Gaussian fitting the line profiles and the overall uncertainty is $\leqslant 10 \%$. Synthetic spectra of giant stars for different input atmospheric parameters and chemical abundances have been computed, using an updated version of the code described in Origlia, Moorwood \& Oliva (1993), which provides full spectral synthesis over the 1-2.5 $\mu \mathrm{m}$ range (see details in Rich \& Origlia (2005) and references therein). The reference solar abundances are from Grevesse \& Sauval (1998). For the observed stars, first guess values of temperature $\left(T_{\text {eff }}\right)$ are derived from optical/IR colors by using the color-temperature transformation of Montegriffo et al. (1998) specifically calibrated on GC giants, gravity $(\log g)$ from theoretical evolutionary tracks according to the location on the Red Giant Branch (RGB), while for the microturbulent velocity $(\xi)$ an average value of $2.0 \mathrm{~km} / \mathrm{s}$ has been adopted (see also Origlia et al. (1997)). Better constraints to stellar parameters are provided by the $\mathrm{CO}$ and $\mathrm{OH}$ line profiles which are very sensitive to their variations, ensuring a good self-consistency of the overall spectral synthesis procedure. Indeed, solutions with stellar parameter variation of $\pm 200 \mathrm{~K}$ in $\mathrm{T}_{\text {eff }}, \pm 0.5$ dex in $\log \mathrm{g}$ and $\pm 0.5 \mathrm{~km} \mathrm{~s}^{-1}$ in $\xi$, and corresponding \pm 0.2 dex abundance 

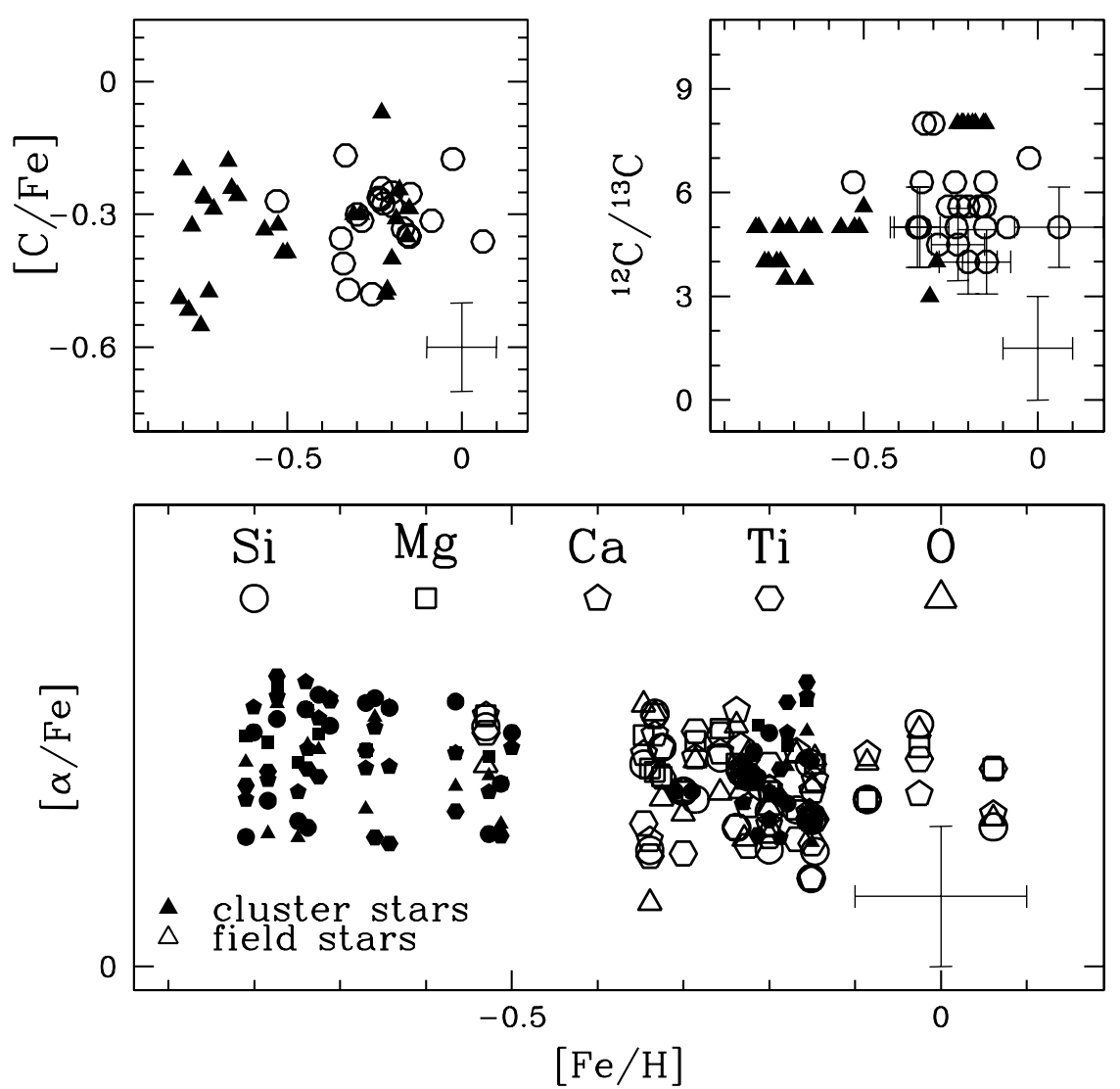

Figure 1. $\alpha$-element over Fe (bottom panel), $[\mathrm{C} / \mathrm{Fe}]$ (top-left panel) and ${ }^{12} \mathrm{C} /{ }^{13} \mathrm{C}$ (top-right panel) abundance ratios as a function of $[\mathrm{Fe} / \mathrm{H}]$ for the observed giants in the Bulge fields (filled symbols) and GCs (empty symbols). Typical errorbars are plotted in the bottom-right corner of each panel.

variations from the best-fitting one are less statistically significant (at $1 \leqslant \sigma \leqslant 3$ level only).

\section{Results and Conclusions}

The abundance and abundance pattern distributions in the cluster and field populations are important in constraining the history of Bulge formation and chemical enrichment McWilliam(1997). Fig. 1 (bottom panel) shows the $[\alpha / \mathrm{Fe}]$ abundance ratios for the various $\alpha$-elements as a function of $[\mathrm{Fe} / \mathrm{H}]$. An overall enhancement is observed both in the Bulge GCs and field populations, in agreement with the optical studies on K giants. No abundance gradients between the Baade's window and the inner bulge field at $\mathrm{l}=0^{\circ}, \mathrm{b}=-1.0^{\circ}$ are found. In the range between $1 / 10$ and solar $[\mathrm{Fe} / \mathrm{H}]$ no systematic trends either with metallicity or among different $\alpha$-elements is observed. It is worth emphasizing that $\mathrm{O}$ behaves like the other $\alpha$-elements, as expected from standard chemical evolution models, but somewhat in contrast with the possible disk-like trend found by Fulbright, Rich \& McWilliam (2004) for their sample of K giants. According to the 
standard scenario of chemical evolution, an overall $[\alpha / \mathrm{Fe}]$ enhancement with respect to solar indicates that the Bulge must have formed from a gas mainly enriched by type II SNe, hence at early epochs and on a relatively short timescale, as the Halo population. From our IR spectra we also derived $\mathrm{Al}$ abundances, finding some Al-Mg anti-correlation (at $\approx 1 \sigma$ ) among the GC giants, while it is absent in the field. This is another interesting similarity with the Halo population (Gratton, Sneden \& Carretta (2004), Sneden et al. (2004)), suggesting that GCs, either in the Halo or in the Bulge could have undergone some self-enrichment process during the early phases of their formation. Finally, in all giants of our survey we measure $\mathrm{C}$ abundances. Fig. 1 (top panels) shows the $[\mathrm{C} / \mathrm{Fe}$ ] and ${ }^{12} \mathrm{C} /{ }^{13} \mathrm{C}$ abundance ratios as a function of $[\mathrm{Fe} / \mathrm{H}] .[\mathrm{C} / \mathrm{Fe}]$ is depleted up to a factor of $\approx 3$ with respect to the solar value, and the ${ }^{12} \mathrm{C} /{ }^{13} \mathrm{C}<10$ isotopic ratio is very low, as normally observed close to the RGB Tip also in metal poor Halo giants (Shetrone (2003), Smith, Terndrup \& Suntzeff (2002), Origlia et al. (2003)). These abundance patterns can be explained with extra-mixing mechanisms due to cool bottom processing which are at work during evolution along the RGB over the full range of metallicity up to solar.

\section{Acknowledgements}

LO and EV acknowledge financial support by the Ministero dell'Istruzione, Università e Ricerca. RMR acknowledges support from grant AST-0098739 from the National Science Foundation. They also acknowledge the Keck Observatory and the NIRSPEC team.

\section{References}

Blanco, V.M., McCarthy, M.F., \& Blanco, B.M. 1984, AJ 89, 636

Carretta, E., Cohen, J., Gratton, R.G., \& Behr, B. 2001, AJ 122, 1469

Frogel, J.A., \& Whitford, A.E. 1987, ApJ 320, 199

Gratton, R., Sneden, C., \& Carretta, E. 2004, ARAA 42, 385

Fulbright, J.P., Rich, R.M., \& McWilliam, A. 2004, AAS 205, 7706

Lee, J., Carney, B. W., \& Balachandran, S. C. 2004, AJ 128, 2388

McLean, I. et al. 1998, SPIE 3354, 566

McWilliam, A., \& Rich, R.M. 1994, ApJS 91, 749

McWilliam, A. 1997, ARAA 35, 503

Melèndez, J., Barbuy, B., Bica, E., Zoccali, M., Ortolani, S., Renzini, A., \& Hill, V. 2003, A\&SA 411,417

Montegriffo, P., Ferraro, F.R., Fusi Pecci, F., \& Origlia, L. 1998, MNRAS 276, 739

Origlia, L., Moorwood, A.F.M., \& Oliva, E. 1993, A\&SA 280536

Origlia, L., Ferraro, F.R., Fusi Pecci, F., \& Oliva, E. 1997, A\&\& 321, 859

Origlia, L., Rich, R.M., \& Castro, S. 2002, AJ 123, 1559

Origlia, L., Ferraro, F.R., Bellazzini, M. \& Pancino, E. 2003, ApJ 591, 916

Origlia, L., \& Rich, R. M. 2004, AJ 127, 3422

Origlia, L., Valenti, E., \& Rich, R.M. 2005, MNRAS 356, 1276

Origlia, L., Valenti, E., Rich, R.M., \& Ferraro, F.R. 2005, MNRAS in press

Rich, R.M., \& Origlia, L. 2005, ApJ in press, astro-ph/0506051

Rich, R.M. 1988, AJ 95, 828

Rich, R.M., \& McWilliam, A. 2000, SPIE 4005, 150

Shetrone, M.d. 2003, ApJ 585, 45

Smith V.V., Terndrup, D.M., \& Suntzeff, N.B. 2002, ApJ 579, 832

Sneden, C., Kraft, R.P., Guhathakurta, P., Peterson, R.C., \& Fulbright, J.P. 2004, AJ 127, 2162

Terndrup, D.M., Frogel, J.A., \& Whitford, A.E. 1990, ApJ 357, 453

Terndrup, D.M., Frogel, J.A., \& Whitford, A.E. 1991, ApJ 378, 742

Zoccali, M., Barbuy, B., Hill, V., Ortolani, S., Renzini, A., Bica, E., Momany, Y., Pasquini, L., Minniti, D., \& Rich, R.M. 2004, A\&A 423, 507 